\title{
Factores de riesgo asociados a la osteoporosis posmenopáusica: Estudio de casos de los Hospitales Vicente Corral y José Carrasco
}

\author{
Sergio Guevara ${ }^{1}$, Astrid Feicán ${ }^{2}$, María del Carmen Ochoa ${ }^{3}$, Carlos Arévalo ${ }^{4}$, María Eugenia \\ Aguirre ${ }^{5}$ \\ ${ }^{1}$ Médico Reumatólogo, Profesor de Reumatología de la Facultad de Ciencias Médicas de la \\ Universidad de Cuenca. \\ ${ }^{2}$ Médico Internista, Profesora de Medicina Interna de la Facultad de Ciencias Médicas de la \\ Universidad de Cuenca, Tratante del Hospital José Carrasco (IESS). \\ ${ }^{3}$ Médico Internista, Tratante del Hospital José Carrasco (IESS), Profesora de la Universidad del \\ Azuay. \\ ${ }^{4}$ Médico, MSH, Director del Hospital de Macas, MSP. \\ ${ }^{5}$ Médico Nutrióloga, Tratante del Hospital Vicente Corral Moscoso. \\ Autor para correspondencia: sergio_guevarap@yahoo.com \\ Fecha de recepción: 27 de febrero 2013 - Fecha de aceptación: 15 de mayo 2013
}

\section{RESUMEN}

El objetivo del estudio es determinar la asociación entre los factores de riesgo: índice de masa corporal bajo, alimentación con bajo contenido de calcio, menarquia tardía, menopausia precoz, sedentarismo y condición socioeconómica de pobreza en pacientes con osteoporosis posmenopáusica (OP) de los Hospitales Vicente Corral y José Carrasco. Estudio de casos y controles, participaron 112 mujeres posmenopáusicas con osteoporosis con densitometría ósea T-score <-2.5 DE y 112 controles, edades comprendidas entre 50 y 65 años, por cada caso se incluyó 1 control pareado por la edad. La muestra se calculó en Epi Info 6, con una prevalencia del 10\% y un nivel de confianza (IC) del 95\%. La edad, índice de masa corporal (IMC), menarquia, menopausia se obtuvieron a través del interrogatorio. La encuesta de tendencia de consumo de calcio, condición socioeconómica y actividad física, se realizaron utilizando instrumentos validados. Los resultados fueron sometidos al cálculo de porcentajes, odds ratio de prevalencia (OR) con un IC95\% y regresión. La media de la ingesta de calcio fue de $597,77 \mathrm{mg}$ por día, el $96 \%$ consumieron menos de $1000 \mathrm{mg}$ de calcio, $17,4 \%$ con condición socioeconómica de pobreza, 79\% de mujeres fueron sedentarias distribuidas en ambos grupos. Existió asociación de osteoporosis con menopausia precoz (OR 4,55 IC95\% 2,23-9,31), condición socioeconómica de pobreza (OR 3,56 IC95\% 1,64-17,73) y menarquia tardía (OR 5,17 IC95\% 1,69-15,81); no hay asociación con bajo IMC, deficiente consumo de calcio y estilo de vida sedentaria.

Palabras clave: Osteoporosis posmenopáusica, factores de riesgo, menarquia, menopausia, sedentarismo, ingesta de calcio.

\begin{abstract}
The purpose of the study presented herein is the assessment of the statistical relation between postmenopausal osteoporosis (PO) and the following risk factors: low body mass index (BMI), low calcium diet, delayed menarche, early menopause, sedentary lifestyle and socioeconomic status. The research involved the screening of 112 postmenopausal women with osteoporosis and 112 control patients, aged 50 to 65 , patients belonging respectively to the Hospitals Vicente Corral and José Carrasco, in Cuenca, Ecuador. A questionnaire addressing calcium-intake, socio-demographic, reproductive and lifestyle-related risk factors was employed previously to bone mineral density and other physical measurements using validated instruments. In addition to the calculation of the mean,
\end{abstract}


standard deviation and percentage of occurrence of the dependent and independent variables, the logistic regression analysis was applied to obtain the odds ratio of prevalence (OR) with a confidence interval (CI) of $95 \%$. Of the examined pop ulation $96 \%$ consume less than $1000 \mathrm{mg}$ of calcium per day and the mean daily calcium intake varies around 597,8 $\mathrm{mg}$. In both groups $17,4 \%$ of the women belong to the socioeconomic status (SES) of poverty, and 79\% are sedentary. OP seems to be related with early menopause osteoporosis (OR 4,55 IC95\% 2,23-9,31), socioeconomic status (OR 3,56 IC95\% 1,64-17,73) and late menarche (OR 5,17 IC95\% 1,69-15,81). OP does not seems to be associated with low BMI, low calcium-intake and sedentary lifestyle.

Keywords: Postmenopausal osteoporosis, risk factors, menarche, menopause, sedentary, calcium intake.

\section{INTRODUCCIÓN}

La osteoporosis posmenopáusica (OP), es una enfermedad metabólica caracterizada por una masa ósea baja con deterioro de la micro arquitectura del tejido óseo, que determina aumento de la fragilidad ósea y riesgo de fractura (Firestein y col., 2009). Tiene una elevada prevalencia, la OP de columna en América Latina es del 12 al 18\% y de fémur proximal entre 8 al 22\%; en mujeres mayores de 50 años y varones mayores de 70 años la prevalencia supera el 30\% (Contreras y col., 2001; Morales-Torres y Gutiérrez-Ureña, 2004; Camargo y col., 2005;). Es un problema de salud pública que afecta a la población mundial. Se estima que más de 200 millones de personas en todo el mundo padecen de OP, 9,4 millones se encuentran en Estados Unidos y ocasiona 1,6 millones de fracturas a nivel mundial, el $50 \%$ de estas fracturas registradas en Europa y Estados Unidos (Arden y Cooper, 1998).

El problema de la OP no sólo es la pérdida de masa ósea sino el riesgo de fractura y las comorbilidades que esto acarrea. La complicación más severa de la osteoporosis es la fractura de cadera, con alta mortalidad (WHO Study Group, 1994; Grados y col., 2004). Es conocido que las pacientes que tienen una fractura en cadera o columna deben permanecer en reposo durante períodos prolongados, lo que produce complicaciones que pueden ser mortales como la embolia grasa que afecta la circulación pulmonar o cardíaca (Lane, 2002).

Los factores de riesgo que contribuyen a la presencia de la OP han sido motivo de controversia entre los que se han señalado la edad, con el envejecimiento se produce mayor pérdida ósea, menor actividad osteoblástica, disminución de la absorción intestinal de calcio, deficiencias nutricionales, carencia de vitamina D y baja exposición solar; además el adulto mayor tiene mayor riesgo de caídas y de sufrir fracturas (Burger y col., 1998; Hermoso de Mendoza, 2003).

La mineralización ósea está relacionada con el índice de masa corporal (IMC), las personas con mayor peso corporal tienen una alta protección contra las fracturas por osteoporosis u otras causas. El mantenimiento de una adecuada composición corporal y peso a lo largo de la vida adulta conseguirán que se alcance la menopausia y la vejez con mejor densidad mineral ósea (Ponce y col., 2002; Oria, 2003). Las pacientes con un IMC bajo $\left(<19 \mathrm{~kg} \mathrm{~m}^{-2}\right)$ tienen menor densidad mineral ósea (DMO) con una deficiente actividad osteoblástica debido a una menor carga mecánica sobre el hueso, con un menor freno de la actividad osteoclástica por una deficiente producción de estrona debido a la falta de panículo adiposo (Dargent-Molina y col., 1996). En el estudio MEDOS, se observó un mayor riesgo de fractura de cadera en personas con un IMC menor de $20 \mathrm{~kg} \mathrm{~m}^{-2}$ (OPS, 2002).

El calcio es un elemento fundamental de la estructura ósea, en el adulto mayor las necesidades de calcio son $1000 \mathrm{mg}$ (Oria, 2003), la ingesta de alimentos ricos en calcio, como vegetales verdes, pescados y derivados lácteos, favorecen que la masa ósea llegue a su máximo nivel en el adulto y permiten disminuir el riesgo de osteoporosis. Es probable que una ingesta de calcio baja durante el período de crecimiento esquelético, pueda disminuir el pico de DMO e incrementar el riesgo de fractura a lo largo de la vida (Lane, 2002). El ejercicio físico tiene un papel importante para el crecimiento y la remodelación del hueso, contribuyendo a la presión y tensión muscular; el sedentarismo afecta el crecimiento y remodelación del hueso por ausencia de estos estímulos y condiciona la posibilidad de desarrollar OP (OPS, 2002). Los pacientes inmovilizados pueden perder 
el $40 \%$ de su masa ósea original en un año, mientras que el estar de pie durante 30 minutos cada día previene la pérdida ósea (Lane, 2002).

La menarquia constituye un importante indicador biológico de la masa ósea futura, mientras más temprano se presente la menarquia, mayor será la masa ósea posterior e inversamente una mujer que presenta una menarquia tardía (> 15 años) desarrolla una menor masa ósea, con déficit de hormonas sexuales femeninas (Hemoso de Mendoza, 2003) que determinan la falta de freno de acción de los osteoclastos lo que provoca una pérdida acelerada del hueso trabecular (Shilbayeh, 2003). La menopausia precoz o cese permanente de la menstruación por pérdida folicular ovárica en mujeres menores de 45 años (Yabur, 2006), disminuye la masa ósea después de la menopausia, con una pérdida del 2 al $4 \%$ anual. El riesgo es mayor cuanto más precoz es la edad de la menopausia. En la menopausia la deprivación estrogénica anula el freno a la acción de los osteoclastos, especialmente a nivel del hueso trabecular (remodelamiento óseo alto).

La limitación de recursos económicos se señala como un factor importante para el desarrollo de diferentes tipos de enfermedades especialmente aquellas que dependen de alimentación adecuada y educación, como la OP; se ha planteado que la condición económica de pobreza, que se obtienen al aplicar instrumentos como el Sistema Integrado de Indicadores Sociales del Ecuador (SIISE), es un factor involucrado en el desarrollo de la enfermedad (SIISE, 2013).

Conociendo que la OP es una enfermedad que puede afectar a un importante sector de la población de la región y el país; es necesario conocer los factores de riesgo que están involucrados en la misma, de allí la importancia de este estudio, que nos permite definir las situaciones que contribuyen a la presencia de la enfermedad y que podrían ser modificables con la intervención. El presente estudio de casos y controles, establece la asociación de la osteoporosis posmenopáusica y factores de riesgo como la edad, IMC bajo, dieta pobre en lácteos, menarquia tardía, menopausia precoz, sedentarismo y condición socioeconómica de pobreza.

\section{MATERIALES Y MÉTODOS}

\subsection{Descripción de la población y los métodos utilizados para la recolección de datos}

Es un estudio de casos y controles, que estableció la asociación de la osteoporosis posmenopáusica y factores de riesgo como la edad, IMC bajo, dieta pobre en lácteos, menarquia tardía, menopausia precoz, sedentarismo y condición socioeconómica de pobreza. Participaron mujeres posmenopáusicas con edades comprendidas entre los 50 y 65 años, que acudieron a la consulta de los Hospitales Vicente Corral y José Carrasco, fueron diagnosticadas de osteoporosis con una densitometría realizada en columna y cadera con resultado de menos -2,5 desviaciones estándar (DE). Por cada caso se incluyó 1 control pareado por la edad. Las restricciones del cálculo de la muestra se realizaron en Stat Calculator, con una prevalencia del 10\%, un nivel de confianza (IC) del $95 \%$, un poder de potencia de $80 \%$, con un odds ratio de prevalencia (OR) de 3. El tamaño de la muestra correspondió a 112 pacientes con osteoporosis y 112 controles.

Los siguientes criterios se utilizan para la composición de la población de la muestra: (i) las mujeres debían residir los últimos 10 años en la ciudad de Cuenca con la edad entre 50 y 65 años y que firmaron el consentimiento informado; y (ii) posmenopáusicas con 1 año de suspensión de los ciclos menstruales. Criterios de exclusión están: (i) pacientes que presentaban alguna enfermedad ósea como la enfermedad de Paget; (ii) mieloma múltiple; (iii) metástasis ósea o que la osteoporosis responda a una enfermedad crónica incapacitante, y (iv) se excluyeron a las pacientes que estaban administrándose fármacos que afecten la densidad mineral ósea.

Si cumplían con los criterios señalados se procedió a realizar la densitometría y la aplicación de los instrumentos para determinar las variables. Fue definido caso de OP por los criterios de la OMS si la densidad mineral ósea (DMO) con dual-energy x-ray absorptiometry (DXA) en cadera o columna 
tiene un T-score ${ }^{1}$ de <-2,5 DE al comparar con la masa ósea de un adulto joven (Melton, 1995; Hermoso de Mendoza, 2003). El equipo utilizado para la medición ósea fue un DXA GE Lunar Prodigy Advance 2005 DXA scanner, utilizando Encore Software 11.40.004, modo de exploración rodilla y columna vertebral.

Una vez cumplido este procedimiento los encuestadores realizaron las preguntas que constan en el formulario previamente elaborado. De las variables en estudio la ocupación se obtuvo de la entrevista, la edad en años cumplidos de la mujer posmenopáusica fue verificada con la cédula de identidad. La variable IMC se obtuvo a partir de la talla del paciente en metro (m) y el peso $(\mathrm{kg})$, utilizando la balanza y tallímetro Marca Seca. Para la medición de la altura la posición de la cabeza estuvo en el plano de Frankfort, con la cara posterior del glúteo y cabeza bien adheridos al plano posterior del estadiómetro. Estos equipos fueron sometidos a control de calidad mensualmente (Mazza, 2003). Se calculó la IMC con el peso (kg) dividido entre estatura en metros al cuadrado (kg $\mathrm{m}^{-2}$ ). Un IMC entre 18,5 y 24,9 se considera normal, y sobre peso un IMC > 24,9.

La dieta con bajo contenido de calcio, se consideró cuando la persona que contestó la encuesta de tendencia de consumo ingería < $1000 \mathrm{mg}$ de calcio por día, para esto se aplicó un instrumento que analiza la ingesta de macro y micronutrientes con el Food Processor II Basic Nutrition and Diet Analysis System, Version 3.13 (Kelly, 1993), que está validado a nivel mundial y ha sido utilizado en otros estudios. La encuesta contenía un registro de 24 horas en 2 días hábiles y un día de fin de semana. Participaron en el levantamiento de los datos estudiantes egresadas en nutrición, las mismas que recibieron un curso de capacitación, utilizaron materiales ilustrativos que permitieron determinar las porciones alimentarias y luego tabularon los datos en el mismo programa.

Tanto la información de la menarquia tardía y menopausia precoz se obtuvieron durante el interrogatorio, se denominó menarquia tardía cuando el primer ciclo menstrual o primera regla ocurrió después de los 15 años y menopausia precoz si el último ciclo menstrual ocurrió en forma natural o quirúrgica antes de los 45 años de edad (Chevalley y col., 2005). Se consideró sedentaria a la persona que no realiza actividad física (caminar, subir escaleras, ejercicio aeróbico, correr, andar en bicicleta, nadar, tenis, fútbol, baloncesto) 3 veces por semana y con una duración de 30 minutos en cada ocasión. No incluyó esta definición a las trabajadoras domésticas ni agricultoras (Ainsworth y col., 2000). Para determinar la actividad física se aplicó el cuestionario internacional de actividad física (IPAC, por sus siglas en inglés) utilizado en Ginebra desde 1998 y ha sido motivo de validez y confiabilidad en varios estudios internacionales (disponible en http://www.ipaq.ki.se/downloads.htm). Este cuestionario hace referencia a la clase de actividad física que las personas hacen como parte de la actividad diaria durante los últimos siete días y consta de cuatro cuestionarios de la actividad física relacionada con el trabajo, transporte, actividades de la casa y recreación, se aplica a personas entre los 15 y 69 años de edad, en el proceso de tabulación se determinó si existe o no sedentarismo.

La condición socioeconómica, se obtuvo aplicando un instrumento a partir de las necesidades básicas insatisfechas que nos da una condición económica de pobreza o no pobreza. Este instrumento fue validado por el Instituto Nacional de Estadística y Censo (INEC). Se estableció como pobreza si presenta dos o más de las siguientes condiciones: (i) la vivienda tiene características físicas inadecuadas (aquellas que son inapropiadas para el alojamiento humano: con paredes exteriores de lata, tela, cartón, estera o caña, plástico u otros materiales de desecho o precario; o con piso de tierra, se incluyen las móviles, refugio natural, puente ó similares); (ii) la vivienda tiene servicios inadecuados (viviendas sin conexión a acueductos o tubería, o sin sanitario conectado al alcantarillado o a pozo séptico); (iii) el hogar tiene una alta dependencia económica (aquellos con más de 3 miembros por persona ocupado y que el Jefe(a) del hogar hubiera aprobado como máximo dos años de educación primaria; (iv) en el hogar existen niños (as) que no asisten a la escuela (al menos un niño de seis a doce años de edad que no asiste a la escuela); (v) el hogar se encuentra en un estado de hacinamiento crítico (aquellos con más de tres personas en promedio por cuarto utilizado para dormir).

\footnotetext{
${ }^{1}$ T-score: El T-score es una comparación de la densidad promedio mineral del hueso (DPH) del paciente con el de una persona sana de 30 años del mismo sexo y etnia.
} 


\subsection{Análisis estadístico}

Una vez recopilada la información se ingresó en una matriz de datos en los programas SPSSTM Versión 15 para Windows ${ }^{\mathrm{TM}}$ y Excel. Se procesó la información con estadística descriptiva e inferencial. Los resultados fueron sometidos al cálculo de porcentajes y odds ratio de prevalencia (OR) con el intervalo de confianza (IC) del 95\%. Las variables discretas: ocupación, actividad física, frecuencia de menarquía tardía, frecuencia de menopausia temprana, frecuencia de deficiencia de calcio, condición socioeconómica y residencia, se analizaron en número de casos (n) y porcentajes (\%); las variables continuas: peso, talla, índice de masa corporal, edad de menarquía, edad de menopausia e ingesta de calcio, en promedio \pm desviación estándar $(\mathrm{X} \pm \mathrm{DE})$.

La distribución de las variables fue analizada con la prueba de Kolmogorov-Smirnov para dos muestras emparejadas. Una diferencia significativa entre la distribución de los casos y los controles no consideró la posibilidad de eliminar valores atípicos ni normalización de la distribución dado el tamaño de la muestra, la selección no aleatoria y el diseño del estudio que por su categoría de investigación epidemiológica observacional no pretendió la verificación de hipótesis al igual que un estudio experimental. El rigor metodológico de pareamiento por una variable, en este diseño, no es para producir grupos comparables en las demás variables. El criterio de pareamiento fue la edad con un rango entre 50 y 65 años, por tanto esta variable no se incluye en el análisis. Para calcular la asociación entre los factores de riesgo y la osteoporosis, explicada como la probabilidad de desarrollar la enfermedad a través de la exposición, utilizamos el OR IC95\%, corroborado por la prueba de Chicuadrado con corrección de Yates en caso de que algún valor observado en la tabla de 2 x 2 tenga una frecuencia menor a 5. Dado que el tamaño de la muestra se calculó con un OR estimado de 3,0 se categorizó la magnitud de los factores de riesgo en función de sus valores.

Para el análisis con regresión logística binomial condicional se consideró como variable dependiente el diagnóstico de osteoporosis que se dicotomizó en presencia (casos) o no (controles) de osteoporosis y se priorizó la variable independiente de más alto OR. Las variables independientes que se incluyeron en el modelo fueron: menarquia tardía, menopausia temprana, condición socioeconómica baja, sedentarismo, índice de masa corporal e ingesta deficiente de calcio. Se fueron eliminando secuencialmente del modelo de regresión las variables de menor significación hasta quedar con variables de OR mayor a la unidad consideradas como factores de riesgo. Para el resultado final se consideraron como variables predictoras de osteoporosis las que tuvieron OR con intervalos de confianza (IC95\%) mayores a la unidad y con un valor $\mathrm{p}<0,05$.

\section{RESULTADOS}

En la caracterización de la población de estudio observamos que $65 \%$ de los casos $(\mathrm{n}=73)$ correspondió a mujeres obreras en tanto que el 50,1\% $(n=57)$ de los controles a empleadas. La frecuencia de fracturas de antebrazo y cuello de fémur, como antecedente, fue similar en los dos grupos; un $21,4 \%$ de los casos tuvo antecedente de fractura de columna. El trabajo remunerado, la actividad física sedentaria, la residencia urbana y el índice de masa corporal, tuvieron una distribución similar. La menarquia tardía $(n=18$ vs $n=4)$ y la menopausia temprana $(n=39$ vs $n=12)$ fueron significativamente mayores en las pacientes con osteoporosis. La condición socioeconómica baja o pobreza fue mayor en las pacientes con osteoporosis 29 (25,8\%) al comparar con los controles. La ingesta deficiente de calcio fue mayor en las mujeres del grupo control que las del grupo con osteoporosis aunque no fueron significativas. Tanto en el grupo con osteoporosis como en el control existió una ingesta de calcio baja en más del 93,8\%. En lo que respecta a la variable actividad física se evidenció sedentarismo en $77,7 \%$ y en $82,1 \%$ de controles.

De acuerdo a la variable condición del peso, la cual fue estructurada en función del peso y la talla para obtener el IMC, el $24,1 \%$ de casos y el $16 \%$ de controles presentaron peso normal, en tanto que el $75,8 \%$ de casos y $83,9 \%$ de controles tuvieron sobrepeso (ver Tabla 1). Los promedios con su desviación estándar y valores mínimos y máximos de las variables independientes: peso, talla, índice de masa corporal y edad de la menarquia fueron similares entre los grupos. Se pudo observar que la 
media del IMC correspondió a sobrepeso en los casos y controles. El promedio de edad de la menopausia fue significativamente menor para las pacientes con osteoporosis.

Tabla 1. Caracterización de 224 mujeres con osteoporosis posmenopáusicas que acuden a la consulta externa de los Hospitales Vicente Corral Moscoso y José Carrasco Arteaga, Cuenca (2007-2008).

\begin{tabular}{lcc}
\hline Características demográficas & $\begin{array}{c}\text { Casos }(\mathrm{n}=112) \\
\mathrm{n}(\%)\end{array}$ & $\begin{array}{c}\text { Controles }(\mathrm{n}=112) \\
\mathrm{n}(\%)\end{array}$ \\
\hline Ocupación & $73(65,2)$ & $55(49,1)$ \\
$\quad$ Obrera & $18(16,1)$ & $27(24,1)$ \\
$\quad$ Empleada privada & $14(12,5)$ & $30(26,8)$ \\
$\quad$ Empleada pública & $15(13,6)$ & $11(9,8)$ \\
Antecedente de fracturas & $8(57,1)$ & $7(87,5)$ \\
$\quad$ Antecedente de fractura de antebrazo & $3(21,4)$ & $1(12,5)$ \\
$\quad$ Antecedente de fractura de cuello de fémur & $3(21,4)$ & - \\
$\quad$ Antecedentes de fractura de columna & $52(46,4)$ & $43(38,4)$ \\
Tiene trabajo remunerado & $87(77,7)$ & $92(82,1)$ \\
Actividad física sedentaria & $29(25,8)$ & $10(8,9)$ \\
Condición socioeconómica baja & $18(16,1)^{*}$ & $4(4,5)$ \\
Menarquia tardía & $39(34,8)^{* *}$ & $12(10,7)$ \\
Menopausia temprana & $105(93,8)$ & $111(99,1)$ \\
Ingesta de calcio deficiente & & \\
Residencia & $104(92,9)$ & $109(97,3)$ \\
$\quad$ Urbana & $8(7,1)$ & $3(2,7)$ \\
Rural & & \\
Índice de masa corporal & $27(24,1)$ & $18(16,07)$ \\
$\quad$ Peso normal & $85(75,8)$ & $94(83,9)$ \\
$\quad$ Sobrepeso & & \\
\hline
\end{tabular}

$* \mathrm{p}=0,011 ; * * \mathrm{p}<0,001$

Tabla 2. Distribución de 224 mujeres posmenopáusicas que acuden a la consulta de los Hospitales Vicente Corral Moscoso y José Carrasco Arteaga, Cuenca (2007-2008) según los factores de riesgo.

\begin{tabular}{|c|c|c|c|c|}
\hline \multirow[t]{2}{*}{ Factores de Riesgo } & $\begin{array}{c}\text { Casos } \\
(\mathrm{n}=112)\end{array}$ & $\begin{array}{l}\text { Controles } \\
(\mathrm{n}=112)\end{array}$ & \multirow[t]{2}{*}{ OR (IC95\%) } & \multirow[t]{2}{*}{ Valor $\mathrm{p}$} \\
\hline & $\mathrm{n}(\%)$ & $\mathrm{n}(\%)$ & & \\
\hline Menarquia tardía & $18(16,07)$ & $4(3,5)$ & $5,17(1,5-18,7)$ & 0,003 \\
\hline Menopausia temprana & $39(34,8)$ & $12(10,7)$ & $4,55(2,2-9.3)$ & 0,001 \\
\hline Condición socioeconómica & $29(25,8)$ & $10(8,9)$ & $3,56(1,5-8,3)$ & 0,001 \\
\hline Peso normal y bajo & $27(24,1)$ & $18(16,07)$ & $1,66(0,8-3,4)$ & 0,133 \\
\hline Ingesta de calcio deficiente & $105(93,7)$ & $111(99,1)$ & $0,14(0,01-1,12)$ & 0,071 \\
\hline Actividad física sedentaria & $87(77,6)$ & $92(82,1)$ & $0,76(0,3-1,5)$ & 0,404 \\
\hline
\end{tabular}

La osteoporosis no mostró relación significativa con el peso de acuerdo a la evaluación Chicuadrado para tabla de contingencia $(\mathrm{p}=0,133)$. En relación a la condición socioeconómica baja se evidenció una asociación significativa $\mathrm{p}=0,001$ (OR 3,56 IC95\% 1,64-17,73), las mujeres pobres presentaron 3,56 veces más riesgo de desarrollar osteoporosis. La ingesta deficiente de calcio en la tabla de contingencia no mostró significancia estadística $\mathrm{p}=0,07$ (IC95\% 0,01-1,17) con corrección de Yates. La edad de la menarquia tardía se relacionó significativamente con la osteoporosis según Chi-cuadrado de contingencia $\mathrm{p}=0,003$ (OR 5,17 IC95\% 1,69-15,81). Se demostró una asociación significativa con la edad de la menopausia, las mujeres con menopausia temprana tuvieron 4,55 veces 
más riesgo de desarrollar osteoporosis $\mathrm{p}=0,001$ (IC95\% 2,33-9,31). Con relación a la osteoporosis y la actividad física no se demuestra asociación significativa por la prueba Chi-cuadrado $p=0,404$ (ver Tabla 2).

En la Tabla 3, presentamos los OR con sus intervalos de confianza y su magnitud de asociación a los factores de riesgo incluidos en el estudio. El análisis por variables individuales otorgó a la menarquia tardía la más alta probabilidad de producir osteoporosis. En orden decreciente, la menopausia temprana y las condiciones socioeconómicas bajas, son factores asociados significativamente. No lo fueron en cambio, el sedentarismo y la ingesta deficiente de calcio.

Tabla 3. Distribución de 224 mujeres posmenopáusicas de los Hospitales Vicente Corral Moscoso y José Carrasco Arteaga (Cuenca, 2007-2008) según análisis de los factores de riesgo por odds.

\begin{tabular}{lccc}
\hline \multirow{2}{*}{ Variable } & \multirow{2}{*}{ OR } & \multicolumn{2}{c}{ IC95\% } \\
\cline { 3 - 4 } & & Inferior & Superior \\
\hline Menarquia tardía & 5,17 & 1,69 & 15,81 \\
Menopausia temprana & 4,55 & 2,23 & 9,31 \\
Condición socioeconómica baja & 3,56 & 1,64 & 17,73 \\
Sedentarismo & 0,75 & 0,39 & 1,45 \\
Índice de masa corporal (sobrepeso) & 0,60 & 0,31 & 1,17 \\
Ingesta deficiente de calcio & 0,13 & 0,01 & 1,17 \\
\hline
\end{tabular}

En un análisis conjunto de los factores de riesgo, aplicando un modelo de regresión logística, la menopausia temprana, la menarquia tardía y las condiciones socioeconómicas bajas, tuvieron en su orden, mayor magnitud de asociación a la osteoporosis. En todos los casos la asociación fue altamente significativa (Tabla 4).

Tabla 4. Distribución de 224 mujeres posmenopáusicas de los Hospitales Vicente Corral Moscoso y José Carrasco Arteaga (Cuenca, 2007-2008) según análisis con regresión logística binomial de los factores de riesgo asociados a osteoporosis.

\begin{tabular}{lcccc}
\hline \multirow{2}{*}{ Variable } & \multirow{2}{*}{ OR } & \multicolumn{2}{c}{ IC95\% } & \multirow{2}{*}{ Valor p } \\
\cline { 3 - 4 } & & Inferior & Superior & \\
\hline Menopausia temprana & 8,28 & 2,74 & 25,06 & 0,0002 \\
Menarquia tardía & 3,56 & 1,21 & 10,48 & 0,0211 \\
Condición socioeconómica baja & 2,87 & 1,30 & 6,34 & 0,0090 \\
\hline
\end{tabular}

\section{DISCUSIÓN}

La osteoporosis es un problema de salud pública que genera preocupación a nivel mundial, especialmente por el riesgo de fractura que presenta todo paciente que padece esta enfermedad (National Institutes of Health, NIH, 2001; Ruiz y Gracia, 2000). Según datos de la OMS el promedio de vida de la población se ha incrementado considerablemente en los últimos años, lo que ha influido en una mayor prevalencia de las enfermedades que se incrementan con la edad como la OP (Jervis $y$ col., 2007). Por lo tanto, se requiere desarrollar investigaciones que nos permitan conocer en nuestro país los principales factores de riesgo asociados a la OP, para implementar medidas de prevención y promoción en la población.

Se han descrito factores de riesgo que son modificables y no modificables (Hakal y col., 2001), entre los modificables tenemos el IMC, en el presente estudio el índice de masa corporal bajo no se asocia con osteoporosis el OR 0,60 IC95\% 0,31-1,17, a diferencia de lo reportado por Hidalgo y Chedrau (2003) quien señaló una asociación del IMC bajo con OP. Frazão y Naveira (2007) de Brasil reportó lo contrario un efecto protector de la OP al sobrepeso con IMC mayor o igual a $30 \mathrm{~kg} \mathrm{~m}^{-2}$ (OR 
0,10 IC95\% 0,05-0,21). Igualmente Barrera y col. (2004) de Chile confirmó el exceso de peso, como efecto protector con IMC entre 30 y $35 \mathrm{~kg} \mathrm{~m}^{-2}$ (OR 0,21 IC95\% 0,11-0,39). En un meta-análisis de Laet y col. (2005), que incluyó 12 estudios prospectivos con seguimiento de 250.000 personas encontraron una asociación de IMC bajo con riesgo de fractura (OR 0,98 IC95\% 0,97-0,99).

La media de ingesta de calcio diaria en esta investigación es de 597,87 mg, superior a la media de ingesta de calcio de $289 \mathrm{mg}$ reportado por Miura y col. (2009) en Filipinas. Al analizar la asociación entre ingesta de calcio y osteoporosis no hay significancia (OR 0,003 IC95\% 0,016-1,11) con corrección de Yates. Esto coincide con un estudio de Varenna y col. (2007), quien no encontró asociación entre la ingesta de calcio y osteoporosis. Sin embargo, en un estudio realizado por Shin $y$ col. (2010) en Korea señaló un promedio de calcio diario de 627 mg por día (OR 0.65 IC95\% 0,430,98) confirmando la asociación de consumo de calcio deficiente con OP. En otro trabajo Masoni $y$ col. (2007) señaló menos riesgo de fractura de cadera si existía una adecuada ingesta de calcio en la juventud.

La media de la edad de la menarquia en el estudio es de 13,4 años; se evidencia asociación entre la menaraquia tardía y la osteoporosis (OR 5,17 IC95\% 1,69-15,81 con un $\mathrm{p}=0,002$ ). Estos resultados se correlacionan con lo reportado por Chevally y col. (2008), quien informó que la edad de la menarquia fue inversamente proporcional al T-score de densidad mineral ósea encontrado en mujeres adultas jóvenes, concluyó que la menarquia tardía es un factor de riesgo para osteoporosis en el brazo. En otro estudio realizado por Shin y col. (2010) encontró que la menarquia tardía se asoció con osteoporosis (OR 1,46 IC95\% 1,14-1,88).

En nuestro estudio la menopausia precoz se asocia con la OP (OR 4,55 IC95\% 2,23-9,31). Un trabajo realizado por Kritz-Silverstein y Barrett-Connor (1993) concluyó que la menopausia temprana causó pérdida ósea a nivel vertebral. Estos resultados coinciden con lo reportado por Sioka y col. (2010), quien encontró correlación entre la osteoporosis y la menopausia precoz. En otro estudio Pinheiro, mencionado en Varenna y col. (2007) señaló como factor de riesgo de la OP a la menopausia precoz (OR 4,12 IC95\% 1,79-9,48). Varenna y col. (2007) confirmó en su análisis modelo lineal que la disminución de la densidad mineral ósea de la columna se asoció con la menopausia temprana $\mathrm{p}=$ 0,001 .

En lo referente a la actividad física en los resultados del presente estudio, tanto en el grupo con osteoporosis como de control predomina el sedentarismo aunque no existe diferencia significativa entre los dos grupos (OR 0,75 IC95\% 0,39-1,45), lo cual difiere con lo publicado por Martine y col. (2009) quien constató que el sedentarismo se asoció con la osteoporosis.

La variable condición socioeconómica de pobreza, que utiliza un instrumento de necesidades básicas insatisfechas del INEC se asocia a la osteoporosis (OR 3,56 IC95\% 1,64-17,73). Una publicación realizada por Demeter y col. (2007) en Canadá, reportó una relación inversa entre estatus socioeconómico y los valores de densidad mineral ósea. Otra investigación sistemática de 11 estudios realizada por Brenman y col. (2010) en Australia señaló una asociación moderada entre las condiciones socioeconómicas deficitarias y las fracturas osteoporóticas.

\section{CONCLUSIONES}

En esta investigación se observa que la osteoporosis en mujeres posmenopáusicas se asocia de manera significativa con los factores de riesgo: menopausia temprana, menarquia tardía y condiciones socioeconómicas bajas, coincidiendo con otros estudios realizados en diversos países. En lo que se refiere a los factores como sedentarismo e ingesta de calcio, no están asociados con la osteoporosis posmenopáusica; sin embargo, conviene destacar que en cuanto al consumo de calcio diario de la población en estudio, el promedio de ingesta es bajo. Estos resultados servirán como elementos de referencia que permitan identificar factores de riesgo asociados a la osteoporosis, intervenir en los mismos si es factible y prevenir el desarrollo de la osteoporosis especialmente en los grupos vulnerables como el adulto mayor, con lo cual podremos evitar fracturas y comorbilidades que implica en muchos casos, limitación funcional e incluso riesgo de muerte por tromboembolias en el paciente incapacitado. Aspiramos que esta investigación sirva de base para futuros estudios. 


\section{AGRADECIMIENTOS}

Agradecemos a la Dirección de Investigación de la Universidad de Cuenca por brindarnos el apoyo financiero para la investigación, al Dr. Jan Feyen por su apoyo en la revisión del artículo, a la Facultad de Ciencias Médicas por el aval académico y apoyo logístico, y a la Tclga. Verónica Ochoa, asistente de investigación, por su aporte técnico y trabajo de campo.

\section{BIBLIOGRAFÍA}

Ainsworth, B.E., D.R. Bassett Jr., S.J. Strath, A.M. Swartz, W.L. O’Brien, R.W. Thompson, D.A. Jones, C.A. Macera, C.D. Kimsey, 2000. Comparison of three methods for measuring the time spent in physical activity. Med. Sci. Sports Exerc., 32, S457-64.

Arden, N., C. Cooper, 1998. Present and future of osteoporosis: epidemiology. In: Meunier, P.J. (Ed.). Osteoporosis: Diagnosis and management. Mosby/Martin Dunitz, London, 1-16.

Barrera, G, D. Bunout, V. Gattás, M.P. de la Maza, L. Leiva, S. Hirsch, 2004. A high body mass index protects against femoral neck osteoporosis in healthy elderly subjects. Nutrition, 20(9), 769-71.

Brennan, S.L., J.A. Pasco, D.M. Urguhart, B. Oldenburg, F. Hanna, A.E. Wluka, 2009. The association between socioeconomic status and osteoporotic fracture en population-based adults: a systematic review. Ostoporosis Int., 21(9), 1623-25.

Buerger, H., C. De Laet, P.L. van Daele, A.E. Weel, J.C. Witteman, A. Hofman, H.A. Pols, 1998. Risk factors for increased bone loss in an ederly population: the Rotterdam Study. Am J. Epidemiol, 1(147), 871-890.

Camargo, M.B., M.S. Cendoroglo, L.R. Ramos, M.R. de Oliveira Latorre, G.L. Saraiva, A. Lage, N.C. Neto, L.M. Araújo, J.G. Vieira, M. Lazarettig-Castro, 2005. Bone mineral density and osteoporosis among predominantly Caucasian elderly population in the city of São Paulo, Brazil. Osteoporos Int., 16(11), 1451-60.

Chakal, F., B. Castelli, J. Durán, G. Medina, 2001. Factores de riesgo y baja masa ósea en la Ciudad de Mérida. Revista de la Facultad de Medicina, 24(2), 181-4.

Chevalley, T., R. Rizzoli, D. Hans, S. Ferrari, J.P. Bonjour, 2005. Interaction between calcium intake and menarcheal age on bone mass gain: an eight-year follow-up study from perpuberty to postmenarche. J. Clin. Endocrinol. Metab., 90(1), 44-51.

Chevalley, T., J..P. Bonjour, S. Ferrari, R. Rizzoli, 2008. Influence of age at menarche on forearm bone microstructure in healthy young women. J. Clin. Endocrinol. Metabol., 93(7), 2594-2601.

Contreras, F., C. Foulloux, A. Bolivar, 2001. Osteoporosis: Factores de riesgo, prevención y tratamiento. Archivos Venezolanos de Farmacología y Terapéutica, 20(1), 27-37.

Demeter, S., W.D. Leslie, L. Lix, L. MacWilliam, G.S. Finlayson, M. Reed, 2007. The effect of socioeconomic status on bone density testing in a public health-care system. Osteoporisis Int., 18(2), 153-8.

Dargent-Molina, P., F. Favier, H. Grandjean, C. Baudoin, A.M. Schott, E. Hausherr, P.J. Meunier, G. Bréat, 1996. Fall-related factors and risk of hip fracture: the EPIDOS prospesctive study. Lancet, 348(9021), 145-9.

Frazão, P., M. Naveira, 2007. Fatores associados à baixa densidade mineral óssea em mulheres brancas. Rev. Saúde Pública, 41(5), 740-8.

Grados F, C. Marcelli, P. Dargent-Molina, C. Roux, J.F. Vergnol, P.J.Meunier, P. Fardellone, 2004. Prevalence of vertebral fractures in French women older than 75 years from the EPIDOS study. Bone, 34, 362-7. 
Hermoso de Mendoza, M.T. Clasificación de la osteoporosis. Factores de riesgo, clínica y diagnóstico diferencial. Anales Sis San Navarra, 26, 1-10.

Hidalgo, L.A., P.A. Chedrau, 2003. Densidad mineral ósea de columna lumbar en una población de mujeres climatéricas ecuatorianas. Rev. Ecuat. Ginecol. Obstet., 10(3), 288-291.

Jervis, R., W. Aguirre, M. Acosta, C. Bracho, S. Córdova, A. Chávez, W. de la Torre, J. González, P. León, E. Naranjo, V.M. Pacheco, R. Rovayo, K. Sánchez, Consenso Ecuatoriano de Osteoporosis 2007. Guías y recomendaciones de manejo diagnóstico y terapéutico de la osteoporosis. Sociedad Ecuatoriana de Metabolismo Mineral (SECUAMEM). En: Palacios, S. (Ed.), Osteoporosis: Prevención, diagnóstico y tratamiento. Sociedad Iberoamericana de Osteología y Metabolismo Mineral (SIBOMM), 41 págs.

Firestein, G.S., R.C. Budd, E.D. Harris, I.B. McInnes, R. Shaun, J.S. Sergent, 2009. Kelley's Textbook of Reumatolog ( $8^{\text {th }}$ ed.), W.B. Saunders Company, 1902 págs.

Kritz-Silverstein, D., E. Barrett-Connor, 1993. Early menopause, number of reproductive years, and bone mineral density in postmenopausal women. Am. J. Public Health, 83(7), 983-8.

De Laet, C. J.A. Kanis, A. Odén, H. Johanson, O. Johnell, P. Delmas, J.A. Eisman, H. Kroger, S. Fujiwara, P. Garnero, E.V. McCloskey, D. Mellstrom, L.J. Melton 3rd, P.J. Meunier, H.A. Pols, J. Reeve, A. Silman, A. Tenenhouse, 2005. Body mass index as a predictor of fracture risk: a meta-analysis. Osteoporosis Int., 16(11), 1330-8.

Kelly, R.B., 1993. The Food Processor II Basic nutrition and diet analysis system, Version 3.13 (1990). J. Fam. Pract., 36(4), pág. 461.

Lane, N.E., 2002. Rheumatic disease clinics of North América. Osteoporosis, 27(1), Ed. Médica Panamericana S.A., Madrid.

Martine, L.A., E.C. Moura, L.C. Santos, D.C. Malta, M.M. Pinhero, 2009. Prevalence of self-reported diagnosis of osteoporosis in Brazil. Rev. Saude Pública, 43 (supl.2), 9 págs.

Masoni, A., M. Morosano, M.F. Tomat, S.M. Pezzotto, A. Sánchez, 2007. Factores de riesgo para osteoporosis y fracturas de cadera Análisis multivariado. Medicina (B. Aires), 67(5), 423-8.

Mazza, J.C., 2003. Mediciones antropométricas. Estandarización de las técnicas de medición, actualizada según parámetros internacionales. PubliCE Standard, Pid: 187.

Melton, L.J., 1995. How many women have osteoporosis now? J. Bone Miner. Res., 10(2), 175-7.

Miura, S., M. Nakamori, M. Yagi, O.L. Saavedra, S. Ikemoto, S. Yamamoto, 2009. Daily calcium intake and physical activity status in urban women living on low incomes in Davao, Philippines: a primary study for osteoporosis prevention. J. Med. Invest., 56(3-4), 130-5.

Morales-Torrres, J., S. Gutiérrez-Ureña, 2004. Osteoporosis Committee of Pan-American League of Associations for Rheumatology. The burden of osteoporosis in Latin America. Osteoporos Int., 15(8), 625-32.

NIH, 2001. Consensus Development Panel on Osteoporosis, Prevention, Diagnosis and Therapy. JAMA, 285(6),785-795.

Oria, E., 2003. Factores preventivos y nutricionales de la osteoporosis. Anales Sis San Navarra, 26(3), 3-18.

OPS, 2002. Módulos de principios de epidemiología para el control de las enfermedades. Unidad 2: Salud y enfermedad en la población ( $2^{\mathrm{a}}$ ed.). Organización Panamericana de la Salud, Washington DC, 64 págs.

Ponce, L., G. Larenas, P. Riedemann, 2002. Alta prevalencia de osteoporosis en mujeres mapuches postmenopáusicas asintomáticas. Rev. méd. Chile, 130(12), 1365-1372.

Ruiz, A., A.G. Gracia, 2000. Osteoporosis: etiopatogenia, clasificación, epidemiología y diagnóstico. En: Blanche i Rubió, J., (Ed.), Manual SER de las Enfermedades Reumáticas (5 ${ }^{\mathrm{a}} \mathrm{ed}$.). Sociedad Española de Reumatología, Editorial Médica Panamericana, pp. 671-9.

Shilbayeh, S., 2003. Prevalence of osteoporosis and its reproductive risk factores among Jordanian women: a cross-sectional study. Osteoporosis Int., 14(11), 929-40. 
Shin, C.S., H.J. Choi, M.J. Kim, J.T. Kim, S.H. Yu, B.K. Koo, H.Y. Cho, S.W. Cho, S.W. Kim, Y.J. Park, H.C. Jang, S.Y. Kim, N.H. Cho, 2010. Prevalence and risk factors of osteoporosis in Korea: a community-based cohort study with lumbar spine and hip bone mineral density. Bone, 47(2), $378-87$.

SIISE, 2013. Sistema Integrado de Indicadores Sociales del Ecuador (SIISE). Descargado de: http://www.siise.gov.ec/PopupFichas.aspx?varl=POBREZA\&var2=ficpob_P05\&var3=1 (condición económica) en 2013.

Sioka, C., A. Fotopoulos, A. Georgiou, X. Xourgia, A. Papadopoulos, J.A. Kalef-Ezra, 2010. Age at menarche, age at menopause and duration of fertility as risk factors for osteoporosis. Climateric, 13(1), 63-71.

Varenna, M., L. Binelli, S. Casari, F. Zucchi, L. Sinigaglia, 2007. Effects of dietary calcium intake on body weight and prevalence of osteoporosis in early posmenopausal women. Am. J. Clin. Nutr., 86(3), 639-44.

WHO Study Group, 1994. Assessment of fracture risk and its application to screening for postmenopausal osteoporosis. World Health Organ. Tech. Rep. Ser., 843, 129 pp.

Yabur, J.A., 2006. La menopausia puesta al día. Gac. Méd. de Caracas, 114(1), 1-12. 